\title{
AVALIAÇÃO DOS EFEITOS DA COBERTURA DE PALHA DE CANA-DE-AÇÚCAR NA UMIDADE E NA PERDA DE ÁGUA DO SOLO
}

\section{JOSÉ G. PERES ${ }^{1}$, CLAUDINEI F. SOUZA ${ }^{2}$, NORBERTO A. LAVORENTI ${ }^{3}$}

RESUMO: No período de 23 de junho a 23 de julho de 2006, nas condições edafoclimáticas da região de Araras - SP, estudou-se a influência da cobertura de palha de cana-de-açúcar colhida mecanicamente nos valores da umidade volumétrica de um Latossolo Vermelho-Escuro, distrófico, A moderado, de textura argilosa. A variação da umidade volumétrica do solo foi avaliada nas camadas de 0 a $0,20 \mathrm{~m}$ e 0,20 a $0,40 \mathrm{~m}$ de profundidade. Os tratamentos consistiram na cobertura do solo com quantidades de palha de cana-de-açúcar equivalentes a 0 e $1,5 \mathrm{~kg} \mathrm{~m}^{-2}\left(0\right.$ e $\left.1,510^{4} \mathrm{~kg} \mathrm{ha}^{-1}\right)$; o primeiro tratamento representando a condição sem palha, e o segundo, a condição com palha. Os resultados coletados na camada de 0-0,20 m mostraram que, no período estudado houve redução dos valores da umidade volumétrica do solo de $0,103 \%$ por dia, na condição com palha, e de $0,223 \%$ por dia, na condição sem palha, mais do que o dobro em relação à primeira condição. Quando se considerou a camada de 0,20-0,40 m, a diminuição dos valores da umidade volumétrica do solo foi bem menor do que a verificada na situação anterior, da ordem de $0,116 \%$ por dia, na condição com palha e de $0,159 \%$ por dia, na condição sem palha. Um ponto importante a ser destacado é que os tratamentos utilizados no experimento tiveram influência decisiva no sentido do movimento da água no solo.

PALAVRAS-CHAVE: cobertura morta, palhada de cana-de-açúcar, umidade do solo.

\section{EVALUATION OF THE EFFECTS OF SUGARCANE STRAW COVERAGE IN MOISTURE AND WATER LOSS OF SOIL}

\begin{abstract}
In the period from June 23 to July 23, 2006, in the regional environment of Araras$\mathrm{SP}$, it was studied the influence of sugarcane straw coverage harvested mechanically in water content of a dystrophic dark-red Latosol, A moderate and clayey texture. The variation of volumetric water content of soil in these plots was assessed in layers of 0 to $0.20 \mathrm{~m}$ and 0.20 to $0.40 \mathrm{~m}$ deep. The experimental treatments consisted of covering the soil with quantities of sugarcane straw equivalent to 0 and $1.5 \mathrm{~kg} \cdot \mathrm{m}^{-2}\left(0\right.$ and $\left.1.5 \times 10^{4} \mathrm{~kg} \cdot \mathrm{ha}^{-1}\right)$, the first representing the condition without straw and the second condition with straw. The results collected in the $0-0.20 \mathrm{~m}$ layer showed that during the study period there was a decrease in soil water content of $0.103 \%$ per day provided with straw and $0.223 \%$ per day without straw, or more than double the water loss recorded previously. When it was considered the soil layer of $0.20-0.40 \mathrm{~m}$, the decrease in soil water content was much lower than that of the previous situation, the order of $0.116 \%$ per day provided with straw and $0.159 \%$ per day without straw. An important point to note is that the treatments used in the experiment had a decisive influence on the direction of water movement in the soil.
\end{abstract}

KEYWORDS: mulching, sugarcane straw, soil moisture.

\section{INTRODUÇÃO}

\footnotetext{
${ }^{1}$ Professor Associado, Departamento de Recursos Naturais e Proteção Ambiental, CCA-UFSCar, Araras - SP, jogepe@ @ca.ufscar.br.

${ }^{2}$ Professor Adjunto, Departamento de Recursos Naturais e Proteção Ambiental, CCA-UFSCar, Araras - SP.

${ }^{3}$ Professor Associado, Departamento de Tecnologia Agroindustral e Socioeconomia Rural, CCA-UFSCar, Araras - SP.

Recebido pelo Conselho Editorial em: 14-10-2009
}

Aprovado pelo Conselho Editorial em: 24-7-2010 
A cultura da cana-de-açúcar tem grande importância socioeconômica para o Brasil e, em particular, para o Estado de São Paulo. Segundo levantamento de safra disponível em CONAB (2008), a produção brasileira de cana-de-açúcar, na safra de2008/09, está estimada em 571,4 milhões de toneladas, que serão colhidas em uma área de 7,0 milhões de hectares. Neste mesmo levantamento, o Estado de São Paulo destaca-se como o maior produtor de cana-de-açúcar do País, com uma produção prevista de 340,5 milhões de toneladas, o que representa $59,6 \%$ da produção nacional. Desse total, 144,0 milhões de toneladas serão destinados à fabricação de açúcar e 196,5 milhões de toneladas para a fabricação de álcool. Nesta safra, a área a ser ocupada pelos canaviais paulistas está estimada em cerca de 3,8 milhões de hectares.

Até o início da década de 1950, não se fazia a queima dos canaviais, que então eram colhidos manualmente. Atualmente, a queima dos canaviais antes da colheita é uma prática amplamente adotada em todas as regiões canavieiras do Brasil e tem como objetivo principal facilitar as operações de corte, carregamento e transporte da cana-de-açúcar.

No entanto, devido aos problemas ambientais que tal prática ocasiona, existe uma forte pressão social para que a agroindústria sucroalcooleira venha abolir esta prática, passando a utilizar a colheita da cana crua. Neste tipo de colheita, a palhada da cana-de-açúcar é deixada quase intacta sobre a superfície do solo quando colhida manualmente ou, então, triturada caso a colheita seja feita mecanicamente.

Os dois tipos de colheita da cana-de-açúcar apresentam vantagens e desvantagens. De acordo com DELGADO (1995), a queima da palhada retira do solo a proteção contra os impactos direto das gotas quando da ocorrência de chuvas intensas, aumenta a sua perda de água por evaporação, reduz a sua capacidade de retenção de água, além de facilitar o desenvolvimento das plantas invasoras. Em contraposição, a sua adoção beneficia as operações de preparo do solo quando da renovação dos canaviais, de cultivo mecânico das socarias, promove o controle da cigarrinha e de outras pragas da cultura, além, é claro, de facilitar a sua colheita, seja ela feita de forma manual, seja mecanizada.

ORLANDO FILHO et al. (1998) e CAMILOTTI et al. (2005) estudaram os efeitos do tipo de colheita - cana crua e cana queimada - nas propriedades físico-químicas de um Latossolo VermelhoEscuro e concluíram que a manutenção da palhada (cana crua) aumentou a capacidade de retenção de água do solo estudado.

Analisando os impactos da palhada da cana-de-açúcar nas propriedades físicas de um solo, TIMM et al. (2002) concluíram que a manutenção da palhada sobre a superfície do solo não afetou nenhum dos seguintes componente do balaço hídrico: escoamento superficial, fluxo de água no solo e capacidade de armazenamento de água.

Considerando-se a importância e a escassez de informações quantitativas inerentes ao tema, esta pesquisa teve como objetivo avaliar os efeitos de uma cobertura de palha de cana-de-açúcar sobre a umidade e as perdas de água do solo.

\section{MATERIAL E MÉTODOS}

Esta pesquisa foi conduzida na área experimental do Centro de Ciências Agrárias - CCA, da Universidade Federal de São Carlos - UFSCar, localizado no município de Araras - SP. As coordenadas geográficas locais são as seguintes: $22^{\circ} 18^{\prime}$ de latitude sul e $47^{\circ} 23^{\prime}$ de longitude oeste. A altitude média da área é de aproximadamente $700 \mathrm{~m}$.

O clima da região, segundo o sistema de Köeppen, é do tipo Cwa, mesotérmico, com verões quentes e úmidos, e invernos secos. As normais climatológicas anuais são as seguintes: precipitação pluvial: $1.414 \mathrm{~mm}$; temperatura: $21,1^{\circ} \mathrm{C}$; evaporação de tanque Classe A: $1.443 \mathrm{~mm}$, velocidade do vento: $1,44 \mathrm{~m} \mathrm{~s}^{-1}$; umidade relativa do ar: $69 \%$ e insolação: 2.573 horas.

O solo predominante na área experimental é classificado como sendo Latossolo Vermelho- 
-Escuro, distrófico, A moderado, de textura argilosa. As características físico-hídricas deste solo foram determinadas pelo Laboratório de Física de Solo do Departamento de Recursos Naturais e Proteção Ambiental do CCA-UFSCar (Tabelas 1 e 2).

TABELA 1. Características físicas do solo da área experimental. Physical characteristics of the experimental area.

\begin{tabular}{|c|c|c|c|c|c|}
\hline \multirow[b]{2}{*}{ Camada (m) } & \multicolumn{3}{|c|}{ Frações Texturais (\%) } & \multicolumn{2}{|c|}{ Massa Específica $\left(\mathrm{kg} \mathrm{m}^{-3}\right)$} \\
\hline & Argila & Silte & Areia & Solo & Partícula \\
\hline $0-0,20$ & 60 & 23 & 17 & 1.270 & 2.970 \\
\hline $0,20-0,40$ & 73 & 14 & 13 & 1.330 & 2.980 \\
\hline
\end{tabular}

TABELA 2. Relação entre o potencial mátrico e a umidade volumétrica do solo da área experimental. Relationship between matrix potential and soil water content of the experimental area.

\begin{tabular}{ccccccccccc}
\hline & \multicolumn{8}{c}{ Potencial Mátrico da Água do Solo $(\mathrm{J} / \mathrm{N}, \mathrm{m})$} \\
\cline { 3 - 10 } & 0 & 1 & 2 & 3 & 5 & 10 & 50 & 100 & 150 \\
\cline { 3 - 10 } Camada $(\mathrm{m})$ & & \multicolumn{7}{c}{ Umidade do solo à base de volume $\left(\theta ; \mathrm{m}^{3} \mathrm{~m}^{-3}\right)$} \\
\cline { 3 - 10 } $0-0,20$ & & 0,574 & 0,400 & 0,369 & 0,314 & 0,304 & 0,300 & 0,278 & 0,255 & 0,231 \\
$0,20-0,40$ & 0,554 & 0,446 & 0,440 & 0,354 & 0,338 & 0,306 & 0,294 & 0,281 & 0,274 \\
\hline
\end{tabular}

Para a determinação da massa específica do solo e para o levantamento da curva de retenção da água no solo, foram utilizadas amostras de solo indeformadas, que foram coletadas por meio de anéis metálicos de $5010^{-6} \mathrm{~m}^{3}\left(50 \mathrm{~cm}^{3}\right)$ de capacidade. As amostras foram coletadas no ponto médio das camadas de solo estudadas, a saber: na profundidade de $0,10 \mathrm{~m}$ na camada de solo de $0-0,20 \mathrm{~m}$, e na profundidade de $0,30 \mathrm{~m}$, na de $0,20-0,40 \mathrm{~m}$.

A análise granulométrica foi realizada pelo método da pipeta (GEE \& BAUDER, 1986; KIEHL, 1979), utilizando como dispersante químico o hidróxido de sódio $(\mathrm{NaOH} ; 1 \mathrm{~N})$. Para a dispersão mecânica da amostra, foi utilizado um agitador rotativo de baixa velocidade ( $35 \mathrm{rpm}$ ), sendo que o tempo de agitação das amostras de solo foi de 16 horas.

A massa específica do solo $\left(\rho_{\mathrm{s}} ; \mathrm{kg} \mathrm{m}^{-3}\right)$ foi determinada utilizando-se das amostras de estruturas indeformadas, descritas anteriormente. A massa específica do solo foi obtida dividindo-se a massa seca da amostra seca em estufa à temperatura de $105^{\circ} \mathrm{C}$, durante 48 horas, pelo volume do anel volumétrico. A massa específica das partículas do solo $\left(\rho_{\mathrm{p}} ; \mathrm{kg} \mathrm{m}^{-3}\right)$ foi determinada utilizandose de picnômetros de $5010^{-6} \mathrm{~m}^{3}\left(50 \mathrm{~cm}^{3}\right)$ de capacidade, previamente calibrados em relação à temperatura. Foram utilizadas amostras de terra fina seca em estufa (TFSE), de aproximadamente $1010^{-3} \mathrm{~kg}(10 \mathrm{~g})$.

As curvas de retenção de água no solo, que relacionam o potencial mátrico da água do solo $(\Psi \mathrm{m})$ com a sua umidade volumétrica $(\theta)$, foram levantadas utilizando-se dos extratores de umidade de Richards (KLUTE, 1986), sendo que, nesta tarefa, foram utilizadas placas porosas para pressões nominais de 0,$1 ; 0,3$ e 1,5 MPa. Para a realização da análise, as amostras indeformadas foram saturadas com água destilada e desaerada, e então submetidas nos extratores de umidade a pressões de 0,$01 ; 0,02 ; 0,03 ; 0,05 ; 0,1 ; 0,5 ; 1$ e $1,5 \mathrm{MPa}$, equivalentes, respectivamente, a potenciais matriciais de $-1 ;-2 ;-3 ;-5 ;-10 ;-50 ;-100$ e $-150 \mathrm{~J} / \mathrm{N}$.

As informações da Tabela 2 foram ajustadas ao modelo proposto por GENUCHTEN (1980), mediante análise de regressão não linear (DOURADO et al., 1990). As equações de ajuste obtidas para as curvas de retenção de água, nas camadas de solo de 0-0,20 m e 0,20-0,40 m, estão apresentadas a seguir: 


$$
\begin{aligned}
& \theta_{0-0,20 m}=0,233+\frac{0,341}{\left[1+(0,0421|\Psi m|)^{1,477}\right]^{0,323}} \\
& \theta_{0,20-0,40 m}=0,272+\frac{0,280}{\left[1+(0,0123|\Psi m|)^{1,752}\right]^{0,429}}
\end{aligned}
$$

em que,

$\theta_{0-0,20 \mathrm{~m}}$ - umidade volumétrica do solo na camada de $0-0,20 \mathrm{~m}\left(\mathrm{~m}^{3} \mathrm{~m}^{-3}\right)$;

$\theta_{0,20-0,40 \mathrm{~m}}$ - umidade volumétrica do solo na camada de $0,20-0,40 \mathrm{~m}\left(\mathrm{~m}^{3} \mathrm{~m}^{-3}\right)$, e

$\Psi \mathrm{m}$ - potencial mátrico da água do solo, $\mathrm{J} / \mathrm{N} ; \mathrm{m}$.

Como durante a realização desta pesquisa foi medida a umidade volumétrica do solo $(\theta)$ e não o potencial mátrico da água do solo $(\Psi \mathrm{m})$, este último foi estimado a partir da manipulação algébrica das eqs.(1) e (2). Pela explicitação do potencial mátrico nestas equações, foram obtidas as seguintes relações funcionais, cujos termos têm os mesmos significados e unidades de medida que nas eqs.(1) e (2):

$$
\begin{aligned}
& \Psi m_{0-0,20 m}=0,238\left(\left(\frac{0,341}{\theta_{0-0,20 m}-0,233}\right)^{3,1}-1\right)^{0,667} \\
& \Psi m_{0,20-0,40 m}=0,813\left(\left(\frac{0,280}{\theta_{0,20-0,40 m}-0,272}\right)^{2,33}-1\right)^{0,571}
\end{aligned}
$$

O potencial gravitacional da água do solo $(\Psi \mathrm{z})$ foi medido considerando-se a superfície do solo como referencial, tendo sido arbitrado que os pontos situados abaixo dela apresentam potencial gravitacional negativo. Assim sendo, para os tratamentos SP10 e CP10, adotou-se o valor -0,10 J/N, enquanto para os tratamentos SP30 e CP30, o valor adotado foi de $-0,30 \mathrm{~J} / \mathrm{N}$. O potencial hidráulico da água do solo $(\Psi \mathrm{h})$ foi determinado somando-se os potenciais mátrico e gravitacional. É importante destacar que todos os valores relativos ao potencial hidráulico, assim como ao mátrico e ao gravitacional, são negativos. Neste estudo, os termos SP e CP correspondem, respectivamente, aos tratamentos sem palha e com palha, enquanto os numerais 10 e 20 correspondem, respectivamente, às profundidades médias das camadas de solo de 0-0,20 m e de 0,20-0,40 m.

O delineamento experimental utilizado no estudo foi o de blocos ao acaso, com os blocos sendo representados pelas médias das avaliações diárias de umidade volumétrica do solo, e os tratamentos, pelas situações de cobertura do solo, a saber: com palha e sem palha. No tratamento com palha, cada parcela experimental recebeu o equivalente a $1,5 \mathrm{~kg} \mathrm{~m}^{-2}\left(1,510^{4} \mathrm{~kg} \mathrm{ha}^{-1}\right) \mathrm{de}$ palhada picada, originária de colheita mecânica da cana crua. $\mathrm{O}$ tratamento sem palha representou a condição de ausência de cobertura do solo. Foram utilizadas oito parcelas experimentais, cada uma delas medindo $1,4 \mathrm{~m}$ por $2 \mathrm{~m}$, sendo que, deste total, quatro parcelas receberam o tratamento com palha, e as quatro outras, o tratamento sem palha.

A umidade volumétrica do solo foi medida por meio de um equipamento de TDR, marca Trase, modelo 6050X1, fabricado pela Soilmoisture Equipament Corp. Para tanto, em cada uma das oito parcelas experimentais, foram instalados dois sensores enterráveis (buriable waveguide), cada um deles medindo 0,20 m de comprimento; um dos sensores prospectando a camada de solo de 0-0,20 m, e o outro, a de 0,20-0,40 m.

Antes do início das medições, a umidade volumétrica do solo na camada de 0-0,20 m foi elevada para um valor médio de $34,3 \%$ ( $\mathrm{s}=1,9 \%$; C.V.=5,7\%), correspondente a um potencial mátrico de aproximadamente $-2,5 \mathrm{~J} / \mathrm{N}$. Na camada de $0,20-0,40 \mathrm{~m}$, estes números foram, respectivamente, de 36,9\% (s=5,6\%; C.V.=8,3\%) e $-3,2 \mathrm{~J} / \mathrm{N}$. Comparando-se estas umidades com aquelas da Tabela 2, pode ser observado que, no início das medições de umidade do solo, as duas camadas de solo estudadas apresentavam umidades próximas da capacidade de campo. 
Para avaliar o desempenho operacional do equipamento de TDR, a umidade volumétrica do solo, no início do experimento, foi também determinada pelo método gravimétrico. Considerandose os 16 pontos amostrados nas duas camadas de solo, a umidade volumétrica média determinada através do TDR foi de 35,6\% ( $\mathrm{s}=2,8 \%$; C.V.=7,2\%), enquanto, no caso do método gravimétrico, este valor foi de $39,4 \%(s=2,8 \%$; C.V.=7,9\%). Embora esta última determinação seja, aproximadamente, $10 \%$ mais elevada do que aquela obtida com o TDR, deve ser salientado que os dois métodos de medição apresentaram boa exatidão, que pode ser atestada pelos coeficientes de variação obtidos. Outro ponto importante a ser destacado nestas determinações é que a sonda avaliou a umidade ao longo de toda a camada, visto que seu sensor tem 0,20 m de comprimento, enquanto no método gravimétrico, a camada avaliada foi de aproximadamente $5 \mathrm{~cm}$, que é o diâmetro aproximado do anel volumétrico utilizado na coleta das amostras de solo. $\mathrm{Na}$ análise estatística dos resultados experimentais, foram utilizados o teste F de Snedecor e o teste $t$ de Student para a comparação das médias dos tratamentos estudados.

\section{RESULTADOS E DISCUSSÃO}

Embora o experimento tenha sido instalado em 23-6-2006, a análise dos resultados foi feita em relação ao período de 27-6-2006 a 23-7-2006. Tal medida pode ser justificada pela necessidade de se ter maior estabilidade nas leituras da umidade do solo pela sonda Trase e, principalmente, porque durante esse período não ocorreu nenhuma chuva. Na Tabela 3, estão apresentados os valores médios da umidade volumétrica do solo para os tratamentos e camadas de solo estudados nesta pesquisa.

TABELA 3. Valores medidos da umidade volumétrica do solo no período de 27-6 a 23-7-2006 (\%). Measured values of soil water content during the period of $6 / 27$ to 7/23/2006 (\%).

\begin{tabular}{|c|c|c|c|c|c|}
\hline Data & Tempo (dia) & SP10 & SP30 & CP10 & $\mathrm{CP} 30$ \\
\hline $27-6-2006$ & 1 & 31,2 & 36,4 & 32,4 & 35,4 \\
\hline $28-6-2006$ & 2 & 30,5 & 36,3 & 31,8 & 34,9 \\
\hline $29-6-2006$ & 3 & 30,1 & 36,0 & 31,7 & 34,6 \\
\hline $30-6-2006$ & 4 & 29,8 & 35,7 & 31,5 & 33,7 \\
\hline $1-7-2006$ & 5 & 30,0 & 35,0 & 31,4 & 33,1 \\
\hline $2-7-2006$ & 6 & 29,6 & 34,9 & 31,5 & 33,3 \\
\hline $3-7-2006$ & 7 & 29,2 & 34,6 & 31,1 & 33,0 \\
\hline 4-7-2006 & 8 & 29,2 & 34,6 & 31,1 & 33,1 \\
\hline $5-7-2006$ & 9 & 29,0 & 34,3 & 31,0 & 32,7 \\
\hline $6-7-2006$ & 10 & 28,6 & 34,1 & 30,9 & 32,8 \\
\hline $7-7-2006$ & 11 & 28,4 & 34,1 & 30,8 & 32,5 \\
\hline $8-7-2006$ & 12 & 27,9 & 33,9 & 30,8 & 32,4 \\
\hline $9-7-2006$ & 13 & 27,8 & 33,9 & 30,7 & 32,5 \\
\hline $10-7-2006$ & 14 & 27,6 & 33,8 & 30,5 & 32,3 \\
\hline $11-7-2006$ & 15 & 27,4 & 33,5 & 30,3 & 32,1 \\
\hline $12-7-2006$ & 16 & 27,4 & 33,4 & 30,6 & 32,3 \\
\hline $13-7-2006$ & 17 & 27,3 & 33,1 & 30,2 & 32,1 \\
\hline $14-7-2006$ & 18 & 27,1 & 33,0 & 30,1 & 32,1 \\
\hline $15-7-2006$ & 19 & 26,6 & 33,1 & 30,0 & 32,2 \\
\hline $16-7-2006$ & 20 & 26,5 & 32,9 & 29,9 & 31,8 \\
\hline $17-7-2006$ & 21 & 26,1 & 32,7 & 29,9 & 31,7 \\
\hline $18-7-2006$ & 22 & 25,9 & 32,8 & 29,7 & 31,9 \\
\hline $19-7-2006$ & 23 & 25,7 & 32,6 & 29,8 & 31,8 \\
\hline $20-7-2006$ & 24 & 25,6 & 32,4 & 29,7 & 31,7 \\
\hline $21-7-2006$ & 25 & 25,5 & 32,2 & 29,4 & 31,6 \\
\hline $22-7-2006$ & 26 & 25,2 & 32,1 & 29,3 & 31,5 \\
\hline $23-7-2006$ & 27 & 25,1 & 32,0 & 29,3 & 31,5 \\
\hline
\end{tabular}


A análise de variância das informações coletadas no experimento (Tabela 3) mostrou que houve diferença estatística altamente significativa $(\mathrm{p}<<0,001)$ entre os tratamentos com e sem palha, tanto na camada de solo de 0-0,20 m quanto na de 0,20-0,40 m, sinalizando que a utilização da palha da cana-de-açúcar como cobertura morta diminui a perda de água no solo.

A variação da umidade volumétrica do solo, na camada $0-0,20 \mathrm{~m}$, considerando-se os dois tratamentos aplicados no solo, pode ser observada na Figura 1. A primeira constatação que nela pode ser feita é que a perda de água nesta profundidade de solo se deu de forma praticamente linear para os dois tratamentos (SP10 e CP30), devendo também ser destacados os altos valores dos coeficientes de determinação das equações de regressão obtidas no ajustamento dos dados.

Também pode ser verificado, pelos coeficientes angulares das respectivas equações de regressão das retas de tendência, que a perda de água no tratamento SP10 (-0,223\% por dia) foi mais do que o dobro da verificada no tratamento CP10 (-0,103\% por dia).

Quantificando-se estes percentuais em termos de perda de água, determinou-se que a perda de água no tratamento sem palha foi de $0,45 \mathrm{~mm} \mathrm{dia}^{-1}$, enquanto no tratamento com palha essa perda foi de $0,21 \mathrm{~mm} \mathrm{dia}^{-1}$, ou seja, houve uma diminuição de 53,3\% na perda de água em decorrência da cobertura do solo. Estes resultados sinalizam que a manutenção da palhada da cana-de-açúcar na superfície do solo é uma prática agrícola muito importante para a conservação da água nas suas camadas superficiais.

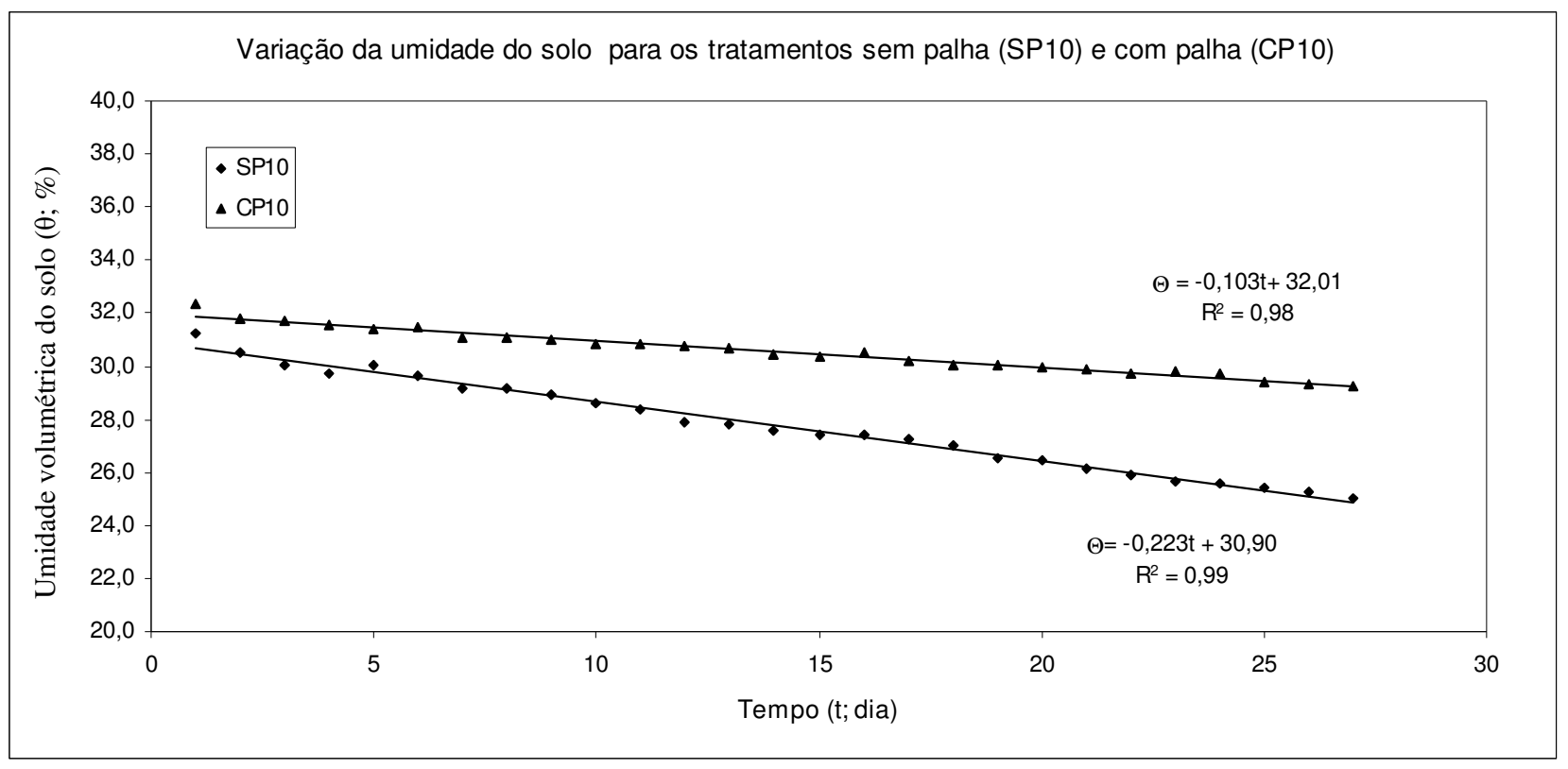

FIGURA 1. Influência da palha da cana-de-açúcar na umidade volumétrica do solo na camada de 0$0,20 \mathrm{~m}$ de profundidade. Influence of sugarcane straw in the soil water content at $\mathbf{0}$ 0,20 m layer.

Na Figura 2, pode ser constatado que a perda de água na camada de solo de 0,20-0,40 m se deu praticamente de forma linear para os dois tratamentos, principalmente a partir do dia 1ํ- 7-2006. 


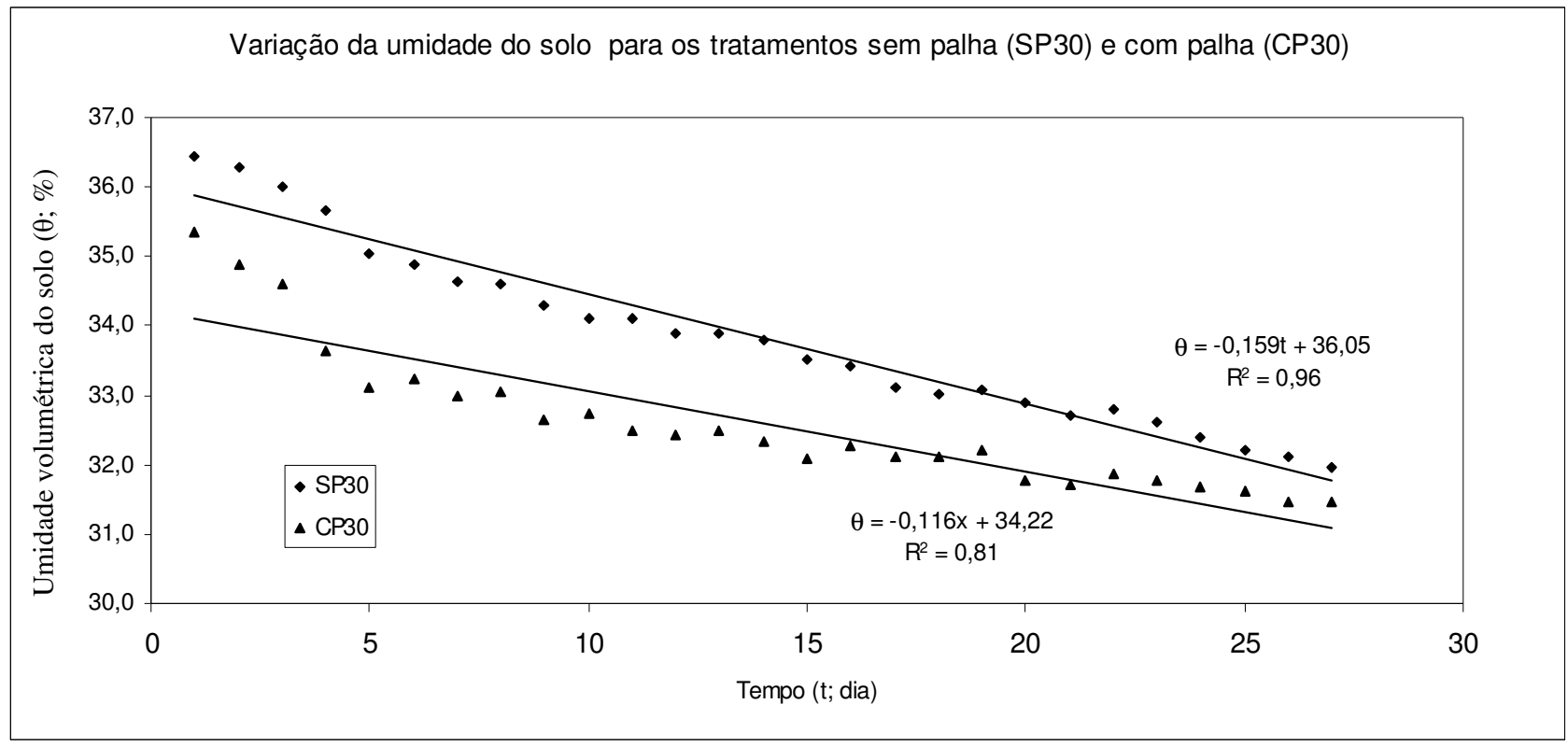

FIGURA 2. Influência da palha da cana-de-açúcar na umidade volumétrica do solo na camada de $0,20-0,40 \mathrm{~m}$ de profundidade. Influence of sugarcane straw in the soil water content at $0,20-0,40 \mathrm{~m}$ layer.

De fato, no caso do tratamento CP30, o melhor ajuste dos dados foi obtido por um modelo polinomial do segundo grau $\left(\mathrm{R}^{2}=0,93\right)$, porém a utilização deste modelo dificultaria a análise dos resultados obtidos sem uma contrapartida de melhora da sua qualidade.

Assim posto, pelos coeficientes angulares das respectivas equações de ajuste das retas de tendência, pode ser verificado que a perda de água no tratamento SP30 (-0,159\% por dia) foi 37,1\% superior àquela do tratamento $\mathrm{CP} 30(-0,116 \%$ por dia), ou seja, ocorreu redução drástica comparativamente aos $116,5 \%$ verificados na camada de solo de 0-0,20 m.

Como feito anteriormente, quantificando-se estes percentuais em termos de perda de água, pode ser determinado que a perda de água no tratamento SP30 foi de $0,32 \mathrm{~mm} \mathrm{dia}^{-1}$, enquanto no tratamento CP30 essa perda foi de $0,23 \mathrm{~mm} \mathrm{dia}^{-1}$, ou seja, houve uma diminuição de $28,1 \%$ na perda de água.

A partir destas informações, pode-se tirar outra conclusão importante, aquela de que a influência da palha no controle da perda de água é diminuída à medida que se aprofunda no perfil do solo.

Na Tabela 4, estão apresentados os valores médios da umidade volumétrica e dos respectivos valores dos potenciais mátrico, gravitacional e hidráulico para os tratamentos SP10 e SP30, os quais são todos negativos. Pelas informações da Tabela 4, pode ser verificado que o potencial hidráulico durante o experimento foi sempre mais negativo no tratamento SP10 do que no SP30, mostrando que, na condição sem palha, a perda de água no solo se faz principalmente por evaporação através da sua superfície, via ascensão capilar.

Também pode ser constatado pelas informações da Tabela 4 que, embora o gradiente de potencial hidráulico ao final do experimento tenha ficado alto $\left(561 \mathrm{~J} / \mathrm{N} / \mathrm{m} ; 561 \mathrm{mca} \mathrm{m}^{-1}\right)$, a umidade do solo permaneceu praticamente constante nos últimos 5 dias do experimento, tanto na camada SP10 quanto na SP30, indicando o cessamento do fluxo de água entre elas, o que se deveu certamente à diminuição exponencial da condutividade hidráulica do solo nessa região, estando de acordo com a lei de Darcy-Buckingham, que descreve o fluxo da água em um solo não saturado (LIBARDI, 2005; JURY \& HORTON, 2004). 
TABELA 4. Valores dos potenciais mátrico $(\Psi \mathrm{m})$, gravitacional $(\Psi \mathrm{z})$ e hidráulico $(\Psi \mathrm{h})$ da água do solo nos tratamentos SP10 e SP30. Values of matrix $(\Psi \mathbf{m})$, gravitational $(\Psi \mathbf{z})$, and hydraulic $(\Psi \mathrm{h})$ potential of soil water in the SP10 and SP30 treatments.

\begin{tabular}{|c|c|c|c|c|c|c|c|}
\hline \multirow[b]{2}{*}{ Data } & & \multicolumn{3}{|c|}{ SP10 } & \multicolumn{3}{|c|}{ SP30 } \\
\hline & $\begin{array}{c}\text { Tempo } \\
\text { (dia) }\end{array}$ & $\begin{array}{l}\Psi \mathrm{m} \\
(\mathrm{J} / \mathrm{N})\end{array}$ & $\begin{array}{c}\mathrm{Iz} \\
(\mathrm{J} / \mathrm{N})\end{array}$ & $\begin{array}{c}\Psi \mathrm{h} \\
(\mathrm{J} / \mathrm{N})\end{array}$ & $\begin{array}{c}\Psi \mathrm{m} \\
(\mathrm{J} / \mathrm{N})\end{array}$ & $\begin{array}{c}\mathrm{z} \\
(\mathrm{J} / \mathrm{N})\end{array}$ & $\begin{array}{c}\mathrm{hh} \\
(\mathrm{J} / \mathrm{N})\end{array}$ \\
\hline $27-6-2006$ & 1 & $-5,0$ & $-0,1$ & $-5,1$ & $-3,4$ & $-0,3$ & $-3,7$ \\
\hline $28-6-2006$ & 2 & $-6,1$ & $-0,1$ & $-6,2$ & $-3,5$ & $-0,3$ & $-3,8$ \\
\hline 29-6-2006 & 3 & $-7,1$ & $-0,1$ & $-7,2$ & $-3,6$ & $-0,3$ & $-3,9$ \\
\hline $30-6-2006$ & 4 & $-7,8$ & $-0,1$ & $-7,9$ & $-3,9$ & $-0,3$ & $-4,2$ \\
\hline $1-7-2006$ & 5 & $-7,1$ & $-0,1$ & $-7,2$ & $-4,3$ & $-0,3$ & $-4,6$ \\
\hline $2-7-2006$ & 6 & $-8,1$ & $-0,1$ & $-8,2$ & $-4,4$ & $-0,3$ & $-4,7$ \\
\hline $3-7-2006$ & 7 & $-9,4$ & $-0,1$ & $-9,5$ & $-4,6$ & $-0,3$ & $-4,9$ \\
\hline $4-7-2006$ & 8 & $-9,6$ & $-0,1$ & $-9,7$ & $-4,7$ & $-0,3$ & $-5,0$ \\
\hline $5-7-2006$ & 9 & $-10,3$ & $-0,1$ & $-10,4$ & $-4,9$ & $-0,3$ & $-5,2$ \\
\hline $6-7-2006$ & 10 & $-11,9$ & $-0,1$ & $-12,0$ & $-5,1$ & $-0,3$ & $-5,4$ \\
\hline $7-7-2006$ & 11 & $-12,8$ & $-0,1$ & $-12,9$ & $-5,1$ & $-0,3$ & $-5,4$ \\
\hline $8-7-2006$ & 12 & $-16,3$ & $-0,1$ & $-16,4$ & $-5,3$ & $-0,3$ & $-5,6$ \\
\hline $9-7-2006$ & 13 & $-16,6$ & $-0,1$ & $-16,7$ & $-5,3$ & $-0,3$ & $-5,6$ \\
\hline $10-7-2006$ & 14 & $-18,5$ & $-0,1$ & $-18,6$ & $-5,4$ & $-0,3$ & $-5,7$ \\
\hline $11-7-2006$ & 15 & $-20,2$ & $-0,1$ & $-20,3$ & $-5,8$ & $-0,3$ & $-6,1$ \\
\hline $12-7-2006$ & 16 & $-20,2$ & $-0,1$ & $-20,3$ & $-5,9$ & $-0,3$ & $-6,2$ \\
\hline $13-7-2006$ & 17 & $-21,6$ & $-0,1$ & $-21,7$ & $-6,4$ & $-0,3$ & $-6,7$ \\
\hline $14-7-2006$ & 18 & $-24,4$ & $-0,1$ & $-24,5$ & $-6,5$ & $-0,3$ & $-6,8$ \\
\hline $15-7-2006$ & 19 & $-33,0$ & $-0,1$ & $-33,1$ & $-6,4$ & $-0,3$ & $-6,7$ \\
\hline $16-7-2006$ & 20 & $-34,6$ & $-0,1$ & $-34,7$ & $-6,7$ & $-0,3$ & $-7,0$ \\
\hline $17-7-2006$ & 21 & $-45,1$ & $-0,1$ & $-45,2$ & $-7,0$ & $-0,3$ & $-7,3$ \\
\hline $18-7-2006$ & 22 & $-51,6$ & $-0,1$ & $-51,7$ & $-6,8$ & $-0,3$ & $-7,1$ \\
\hline $19-7-2006$ & 23 & $-65,1$ & $-0,1$ & $-65,2$ & $-7,2$ & $-0,3$ & $-7,5$ \\
\hline $20-7-2006$ & 24 & $-68,1$ & $-0,1$ & $-68,2$ & $-7,5$ & $-0,3$ & $-7,8$ \\
\hline $21-7-2006$ & 25 & $-78,5$ & $-0,1$ & $-78,6$ & $-8,0$ & $-0,3$ & $-8,3$ \\
\hline $22-7-2006$ & 26 & $-99,0$ & $-0,1$ & $-99,1$ & $-8,2$ & $-0,3$ & $-8,5$ \\
\hline $23-7-2006$ & 27 & $-120,9$ & $-0,1$ & $-121,0$ & $-8,5$ & $-0,3$ & $-8,8$ \\
\hline
\end{tabular}

Na Figura 3, os valores em módulo dos potenciais hidráulicos para os tratamentos SP10 e SP30 estão plotados em relação ao tempo. Um ponto importante, que pode ser bem visualizado na Figura 3, é que o potencial hidráulico no tratamento SP30 variou pouco durante o experimento (mínimo de $-3,7$ e máximo de $-10,1 \mathrm{~J} / \mathrm{N}$ ), além de mostrar que a sua diminuição se deu de forma praticamente linear. O mesmo, entretanto, não aconteceu no tratamento SP10, no qual o potencial hidráulico variou ao longo do experimento (mínimo de $-5,1$ e máximo de $-121,0 \mathrm{~J} / \mathrm{N}$ ), além do que a sua diminuição se deu de forma praticamente exponencial, o que certamente se deveu à exposição da superfície do solo aos efeitos diretos da radiação solar e do vento. Destaque-se, ainda, que o solo no tratamento SP10 está próximo do seu ponto de murchamento permanente, enquanto em SP30 ele se encontra pouco acima da capacidade de campo.

$\mathrm{Na}$ Tabela 5, estão apresentados os valores médios da umidade volumétrica e dos respectivos valores dos potenciais mátrico, gravitacional e hidráulico para os tratamentos CP10 e CP30, os quais são todos negativos. Também pode ser verificado que o potencial hidráulico durante o experimento foi sempre mais negativo no tratamento CP30 do que no CP10, sinalizando que, na condição com palha, a perda de água no solo se faz principalmente por percolação profunda, via infiltração.

Esta evidência experimental mostra que a presença da palhada na superfície do solo diminui os efeitos da radiação solar, que não se faz sentir com tanta intensidade quanto no tratamento alternativo, diminuindo substancialmente as perdas de água por evaporação. 


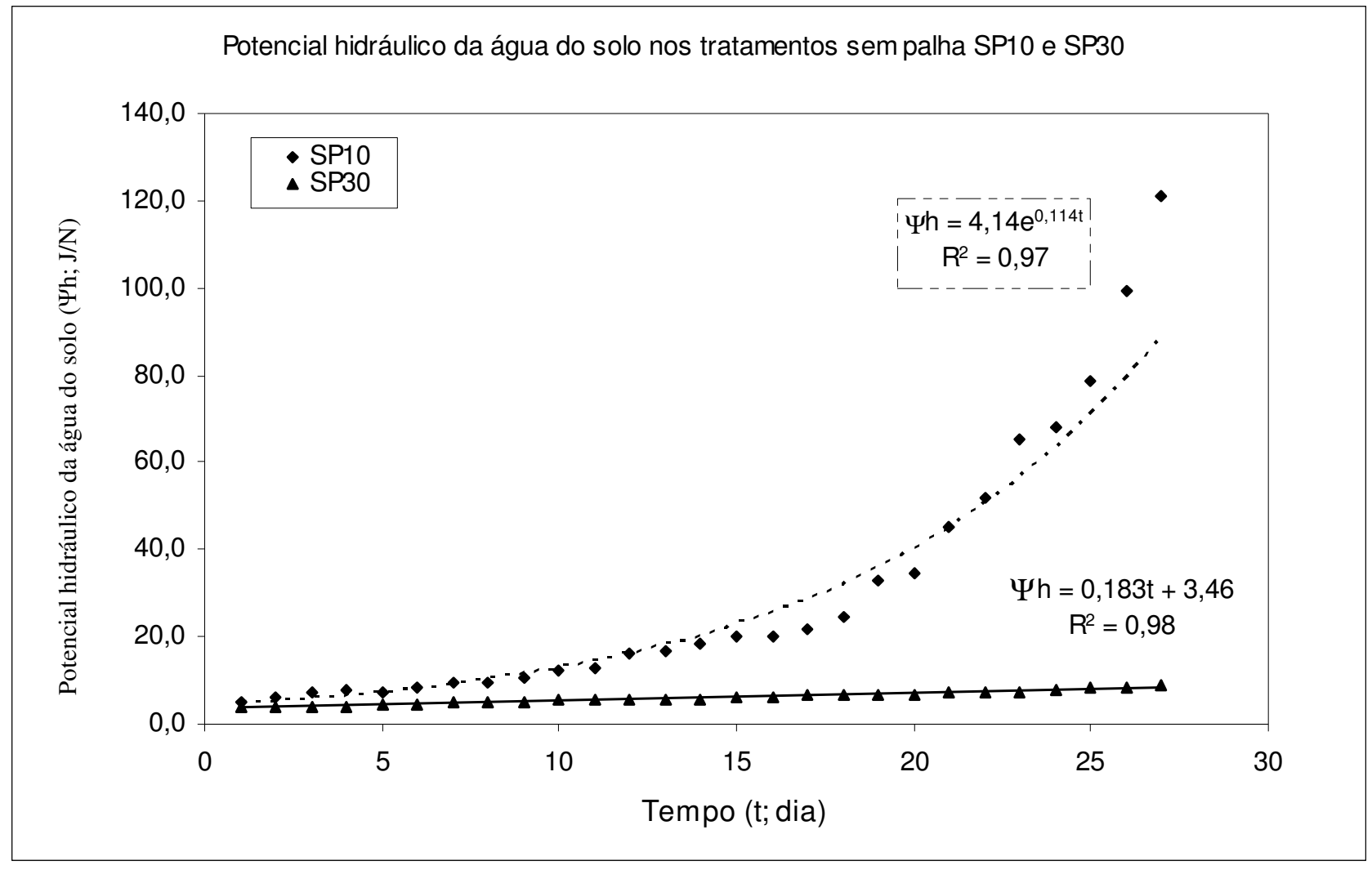

FIGURA 3. Variação do potencial hidráulico da água do solo nos tratamentos SP10 e SP30. Variation of hydraulic potential of soil water in the SP10 and SP30 treatments.

É importante destacar que, em razão de os valores dos potenciais hidráulicos serem próximos nos dois tratamentos, os gradientes de potencial hidráulico são pequenos, principalmente na fase final do experimento (4 J/N/m; 4 mca m$^{-1}$ ), resultando, pela lei de Darcy-Buckingham, em perda de água menor do que aquela verificada no tratamento sem palha.

$\mathrm{Na}$ Figura 4, os valores em módulo dos potenciais hidráulicos para os tratamentos CP10 e CP30 estão plotados em relação ao tempo. Nesta figura, pode ser observado que o potencial hidráulico nos dois tratamentos estudados (CP10 e CP30) diminuiu de forma praticamente linear nos dois casos, assim como também ocorreu no tratamento SP30, indicando que a cobertura de palha praticamente inibe os efeitos da radiação solar e do vento na superfície do solo.

Outro ponto que também pode ser visualizado é que o gradiente de potencial hidráulicos se manteve praticamente constante ao longo do experimento, uma vez que as retas de ajustamento dos dados apresentam coeficientes angulares praticamente iguais. Como durante o experimento o gradiente de potencial hidráulico entre $\mathrm{CP} 10$ e $\mathrm{CP} 30$ foi pequeno (média=6,9 J/N/m; s=2,5 J/N/m; C.V.=35,4\%), esta é a principal razão para a diminuição do fluxo de água e, segundo a lei de DarcyBuckingham, da menor perda de água verificada no tratamento com palha. 
TABELA 5. Valores dos potenciais mátrico $(\Psi \mathrm{m})$, gravitacional $(\Psi \mathrm{z})$ e hidráulico $(\Psi \mathrm{h})$ da água do solo nos tratamentos CP10 e CP30. Values of matrix $(\Psi \mathbf{m})$, gravitational $(\Psi \mathbf{z})$, and hydraulic ( $\Psi \mathrm{h})$ potential of soil water in the CP10 and CP30 treatments.

\begin{tabular}{|c|c|c|c|c|c|c|c|}
\hline \multirow[b]{2}{*}{ Data } & \multirow[b]{2}{*}{$\begin{array}{c}\text { Tempo } \\
\text { (dia) }\end{array}$} & \multicolumn{3}{|c|}{ CP10 } & \multicolumn{3}{|c|}{ CP30 } \\
\hline & & $\begin{array}{c}\Psi \mathrm{m} \\
(\mathrm{J} / \mathrm{N})\end{array}$ & $\begin{array}{c}\Psi_{\mathrm{z}} \\
(\mathrm{J} / \mathrm{N})\end{array}$ & $\begin{array}{l}\Psi \mathrm{h} \\
(\mathrm{J} / \mathrm{N})\end{array}$ & $\begin{array}{c}\Psi \mathrm{m} \\
(\mathrm{J} / \mathrm{N})\end{array}$ & $\begin{array}{c}\Psi \mathrm{z} \\
(\mathrm{J} / \mathrm{N})\end{array}$ & $\begin{array}{c}\Psi \mathrm{h} \\
(\mathrm{J} / \mathrm{N})\end{array}$ \\
\hline 27-6-2006 & 1 & $-3,8$ & $-0,1$ & $-3,9$ & $-4,1$ & $-0,3$ & $-4,4$ \\
\hline $28-6-2006$ & 2 & $-4,3$ & $-0,1$ & $-4,4$ & $-4,4$ & $-0,3$ & $-4,7$ \\
\hline $29-6-2006$ & 3 & $-4,4$ & $-0,1$ & $-4,5$ & $-4,7$ & $-0,3$ & $-5,0$ \\
\hline $30-6-2006$ & 4 & $-4,7$ & $-0,1$ & $-4,8$ & $-5,6$ & $-0,3$ & $-5,9$ \\
\hline $1-7-2006$ & 5 & $-4,8$ & $-0,1$ & $-4,9$ & $-6,3$ & $-0,3$ & $-6,6$ \\
\hline $2-7-2006$ & 6 & $-4,8$ & $-0,1$ & $-4,9$ & $-6,1$ & $-0,3$ & $-6,4$ \\
\hline $3-7-2006$ & 7 & $-5,2$ & $-0,1$ & $-5,3$ & $-6,5$ & $-0,3$ & $-6,8$ \\
\hline $4-7-2006$ & 8 & $-5,2$ & $-0,1$ & $-5,3$ & $-6,4$ & $-0,3$ & $-6,7$ \\
\hline $5-7-2006$ & 9 & $-5,3$ & $-0,1$ & $-5,4$ & $-7,1$ & $-0,3$ & $-7,4$ \\
\hline $6-7-2006$ & 10 & $-5,5$ & $-0,1$ & $-5,6$ & $-6,9$ & $-0,3$ & $-7,2$ \\
\hline $7-7-2006$ & 11 & $-5,6$ & $-0,1$ & $-5,7$ & $-7,4$ & $-0,3$ & $-7,7$ \\
\hline $8-7-2006$ & 12 & $-5,7$ & $-0,1$ & $-5,8$ & $-7,5$ & $-0,3$ & $-7,8$ \\
\hline $9-7-2006$ & 13 & $-5,8$ & $-0,1$ & $-5,9$ & $-7,4$ & $-0,3$ & $-7,7$ \\
\hline $10-7-2006$ & 14 & $-6,3$ & $-0,1$ & $-6,4$ & $-7,7$ & $-0,3$ & $-8,0$ \\
\hline $11-7-2006$ & 15 & $-6,5$ & $-0,1$ & $-6,6$ & $-8,2$ & $-0,3$ & $-8,5$ \\
\hline $12-7-2006$ & 16 & $-6,1$ & $-0,1$ & $-6,2$ & $-7,8$ & $-0,3$ & $-8,1$ \\
\hline $13-7-2006$ & 17 & $-6,8$ & $-0,1$ & $-6,9$ & $-8,2$ & $-0,3$ & $-8,5$ \\
\hline $14-7-2006$ & 18 & $-7,0$ & $-0,1$ & $-7,1$ & $-8,2$ & $-0,3$ & $-8,5$ \\
\hline $15-7-2006$ & 19 & $-7,1$ & $-0,1$ & $-7,2$ & $-8,0$ & $-0,3$ & $-8,3$ \\
\hline $16-7-2006$ & 20 & $-7,4$ & $-0,1$ & $-7,5$ & $-9,0$ & $-0,3$ & $-9,3$ \\
\hline $17-7-2006$ & 21 & $-7,5$ & $-0,1$ & $-7,6$ & $-9,1$ & $-0,3$ & $-9,4$ \\
\hline $18-7-2006$ & 22 & $-7,9$ & $-0,1$ & $-8,0$ & $-8,7$ & $-0,3$ & $-9,0$ \\
\hline $19-7-2006$ & 23 & $-7,7$ & $-0,1$ & $-7,8$ & $-9,0$ & $-0,3$ & $-9,3$ \\
\hline $20-7-2006$ & 24 & $-7,9$ & $-0,1$ & $-8,0$ & $-9,2$ & $-0,3$ & $-9,5$ \\
\hline $21-7-2006$ & 25 & $-8,7$ & $-0,1$ & $-8,8$ & $-9,4$ & $-0,3$ & $-9,7$ \\
\hline $22-7-2006$ & 26 & $-9,1$ & $-0,1$ & $-9,2$ & $-9,8$ & $-0,3$ & $-10,1$ \\
\hline 23-7-2006 & 27 & $-9,2$ & $-0,1$ & $-9,3$ & $-9,8$ & $-0,3$ & $-10,1$ \\
\hline
\end{tabular}




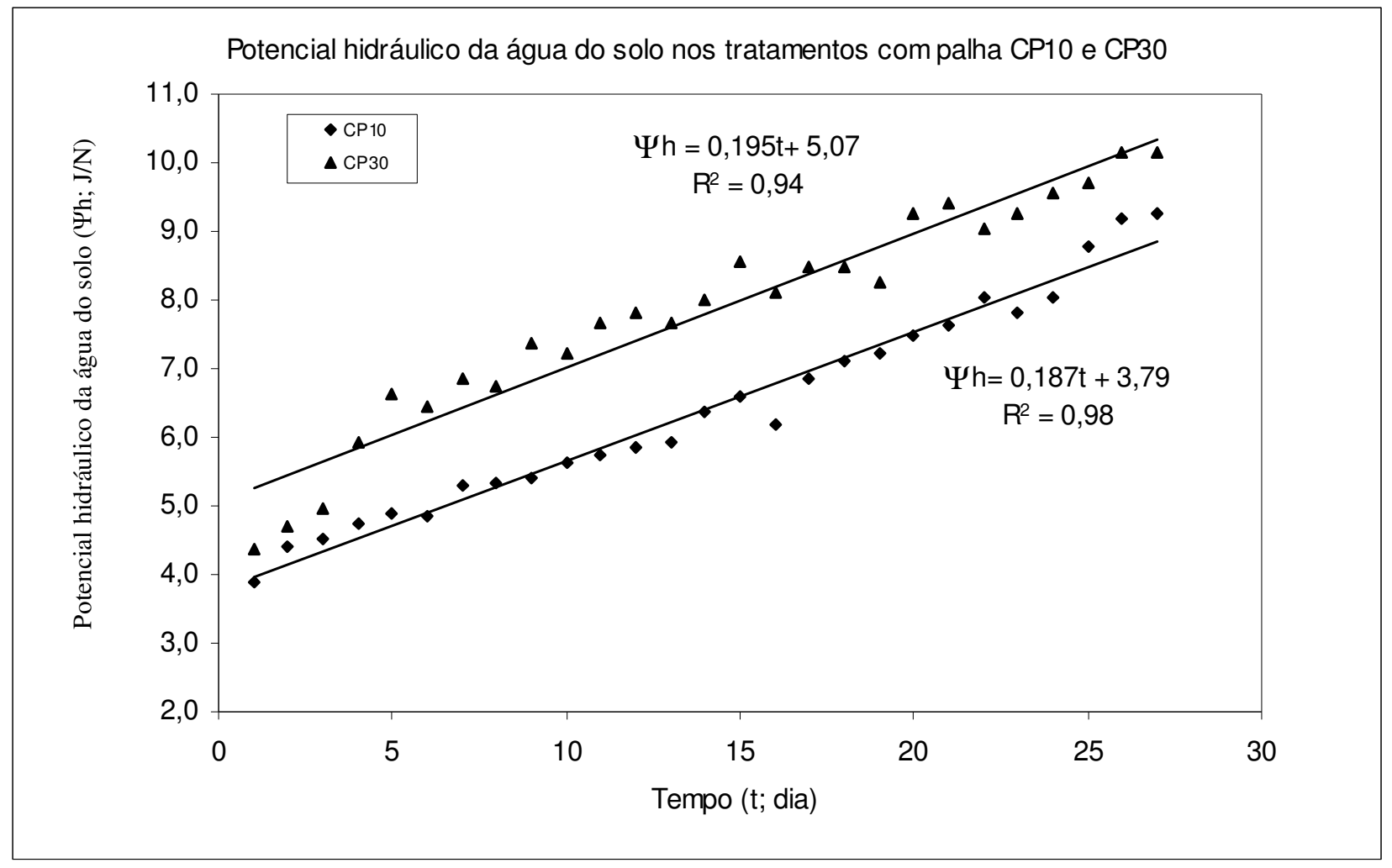

FIGURA 4. Variação do potencial hidráulico da água do solo nos tratamentos com palha: CP10 e CP30. Variation of hydraulic potential of soil water in the CP10 and CP30 treatments.

\section{CONCLUSÕES}

Na camada de 0-0,20 m, a presença da palha da cana-de-açúcar na superfície do solo reduziu as perdas de água praticamente à metade daquela verificada na condição de solo descoberto.

A influência da cobertura de palha da cana-de-açúcar na umidade volumétrica do solo diminui com o aumento da profundidade do solo. 3. A perda de água no solo na condição sem palha se deu praticamente por evaporação, enquanto na condição com palha a perda de água ocorreu preponderantemente por percolação profunda.

\section{REFERÊNCIAS}

CAMILOTTI, F.; ANDRIOLI, I.; DIAS, F.L.F.; CASAGRANDE, A.A.; SILVA, A.R.; MUTTON, M.A.; CENTURION, J.F. Efeito prolongado de sistemas de preparo do solo com e sem cultivo de soqueira de cana crua em algumas propriedades físicas do solo. Engenharia Agrícola, Jaboticabal, v.25, n.1, p.189-198, 2005.

CONAB. COMPANHIA NACIONAL DE ABASTECIMENTO. Acompanhamento da safra brasileira de cana-de-açúcar, safra 2008/2009, terceiro levantamento. Brasília, 2008. 18 p.

DELGADO, A.A. Os efeitos da queima dos canaviais. STAB: Açúcar, Álcool e Subprodutos, Piracicaba, v.3, n.1, p.42-45, 1995.

DOURADO NETO, D.; LIER, Q. J. van; BOTREL, T. A.; LIBARDI, P. L. Programa para a confecção da curva de retenção da água no solo utilizando o modelo de van Genuchten. Engenharia Rural, Piracicaba, v.1, p.92-102, 1990. 
GEE, G.W.; BAUDER, J.W. Particle-size analysis. In: KLUTE, A. (Ed.). Methods of soil analysis: physical and mineralogical methods. $2^{\text {nd }}$ ed. Madison: American Society Agronomy, 1986. v.1. p.383-411.

GENUCHTEN, M.T. van. A closed form equation for predicting the hydraulic conductivity of unsaturated soils. Soil Science Society America Journal, Madison, v.44, p.892-898, 1980.

JURY, W.A.; HORTON, R. Soil physics. $6^{\text {th }}$ ed. New Jersey: John Wiley \& Sons, 2004. 370 p. KIEHL, E.L. Manual de edafologia. São Paulo: Ceres, 1979. 262 p.

KLUTE, A. Water retention: laboratory methods. In: KLUTE, A. (Ed.) Methods of soil analysis. Physical and mineralogical methods. $2^{\text {nd }}$ ed. Madison: American Society Agronomy, 1986. Part I. p.635-662.

LIBARDI, P.L. Dinâmica da água no solo. São Paulo: Edusp, 2005. 335 p. (Acadêmica, 61)

ORLANDO FILHO, J.; ROSSETO, R.; MURAOKA, T.; ZOTELLI, H.B. Efeitos do sistema de despalha (cana crua x cana queimada) sobre algumas propriedades do solo. STAB: Açúcar, Álcool e Subprodutos, Piracicaba, v.16, p.30-33, 1998.

TIMM, L.C.; OLIVEIRA, J.C.M.; TOMINAGA, T.T.; CÁSSARO, F.A.M.; REICHARDT, K.; BACCHI; O.O.S. Water balance of a sugarcane crop: quantitative and qualitative aspects of its measurement. Revista Brasileira de Engenharia Agrícola e Ambiental, Campina Grande, v.6, p.57$62,2002$. 